(C) 2017 IEEE. Personal use of this material is permitted. Permission from IEEE must be obtained for all other uses, in any current or future media, including reprinting/republishing this material for advertising or promotional purposes, creating new collective works, for resale or redistribution to servers or lists, or reuse of any copyrighted component of this work in other works.

https://doi.org/10.1109/ICCV.2017.117 


\title{
Large Pose 3D Face Reconstruction from a Single Image via Direct Volumetric CNN Regression
}

\author{
Aaron S. Jackson ${ }^{1} \quad$ Adrian Bulat $^{1} \quad$ Vasileios Argyriou $^{2} \quad$ Georgios Tzimiropoulos $^{1}$ \\ ${ }^{1}$ The University of Nottingham, UK $\quad{ }^{2}$ Kingston University, UK \\ ${ }^{1}$ \{aaron.jackson, adrian.bulat, yorgos.tzimiropoulos\}@nottingham.ac.uk \\ 2 vasileios.argyriou@ kingston.ac.uk
}
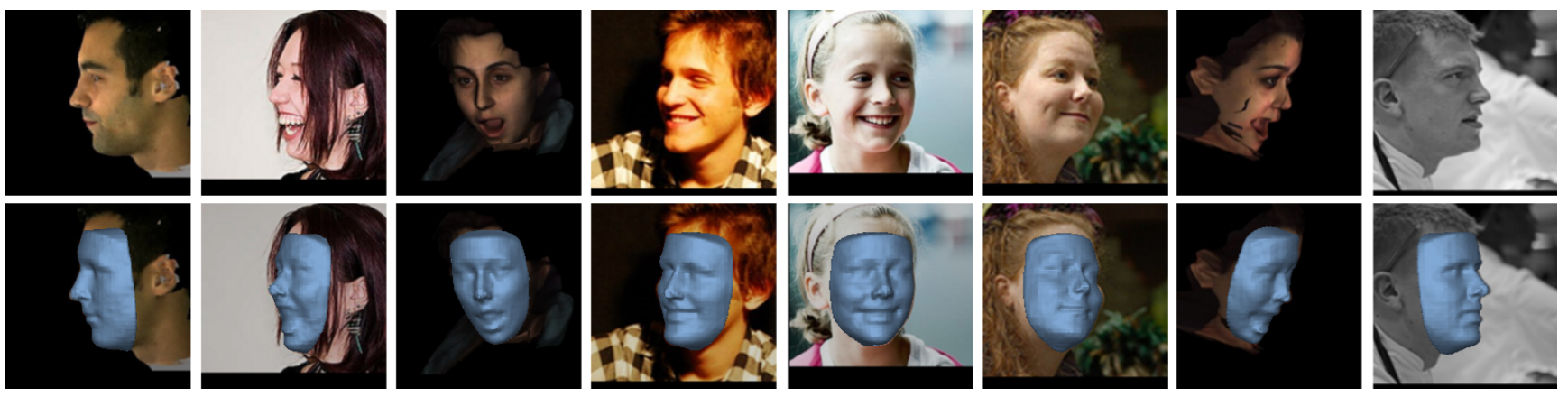

Figure 1: A few results from our $V R N$ - Guided method, on a full range of pose, including large expressions.

\begin{abstract}
$3 D$ face reconstruction is a fundamental Computer Vision problem of extraordinary difficulty. Current systems often assume the availability of multiple facial images (sometimes from the same subject) as input, and must address a number of methodological challenges such as establishing dense correspondences across large facial poses, expressions, and non-uniform illumination. In general these methods require complex and inefficient pipelines for model building and fitting. In this work, we propose to address many of these limitations by training a Convolutional Neural Network (CNN) on an appropriate dataset consisting of $2 D$ images and $3 D$ facial models or scans. Our CNN works with just a single $2 D$ facial image, does not require accurate alignment nor establishes dense correspondence between images, works for arbitrary facial poses and expressions, and can be used to reconstruct the whole $3 D$ facial geometry (including the non-visible parts of the face) bypassing the construction (during training) and fitting (during testing) of a 3D Morphable Model. We achieve this via a simple CNN architecture that performs direct regression of a volumetric representation of the $3 D$ facial geometry from a single $2 D$ image. We also demonstrate how the related task
\end{abstract}

of facial landmark localization can be incorporated into the proposed framework and help improve reconstruction quality, especially for the cases of large poses and facial expressions. Our training code for our unguided method, pretrained models and the voxelised training data is all available at http: //aaronsplace.co.uk

\section{Introduction}

3D face reconstruction is the problem of recovering the 3D facial geometry from 2D images. Despite many years of research, it is still an open problem in Vision and Graphics research. Depending on the setting and the assumptions made, there are many variations of it as well as a multitude of approaches to solve it. This work is on 3D face reconstruction using only a single image. Under this setting, the problem is considered far from being solved. In this paper, we propose to approach it, for the first time to the best of our knowledge, by directly learning a mapping from pixels to $3 \mathrm{D}$ coordinates using a Convolutional Neural Network (CNN). Besides its simplicity, our approach works with totally unconstrained images downloaded from the Web, including facial images of arbitrary poses, facial expressions and occlusions, as shown in Fig. 1. 
Motivation. No matter what the underlying assumptions are, what the input(s) and output(s) to the algorithm are, 3D face reconstruction requires in general complex pipelines and solving non-convex difficult optimization problems for both model building (during training) and model fitting (during testing). In the following paragraph, we provide examples from 5 predominant approaches:

1. In the 3D Morphable Model (3DMM) [2, 20], the most popular approach for estimating the full 3D facial structure from a single image (among others), training includes an iterative flow procedure for dense image correspondence which is prone to failure. Additionally, testing requires a careful initialisation for solving a difficult highly non-convex optimization problem, which is slow.

2. The work of [10], a popular approach for $2.5 \mathrm{D}$ reconstruction from a single image, formulates and solves a carefully initialised (for frontal images only) non-convex optimization problem for recovering the lighting, depth, and albedo in an alternating manner where each of the sub-problems is a difficult optimization problem per se.

3. In [11], a quite popular recent approach for creating a neutral subject-specific $2.5 \mathrm{D}$ model from a near frontal image, an iterative procedure is proposed which entails localising facial landmarks, face frontalization, solving a photometric stereo problem, local surface normal estimation, and finally shape integration.

4. In [23], a state-of-the-art pipeline for reconstructing a highly detailed 2.5D facial shape for each video frame, an average shape and an illumination subspace for the specific person is firstly computed (offline), while testing is an iterative process requiring a sophisticated pose estimation algorithm, 3D flow computation between the model and the video frame, and finally shape refinement by solving a shape-from-shading optimization problem.

5. More recently, the state-of-the-art method of [21] that produces the average (neutral) 3D face from a collection of personal photos, firstly performs landmark detection, then fits a $3 \mathrm{DMM}$ using a sparse set of points, then solves an optimization problem similar to the one in [11], then performs surface normal estimation as in [11] and finally performs surface reconstruction by solving another energy minimisation problem.

Simplifying the technical challenges involved in the aforementioned works is the main motivation of this paper.

\subsection{Main contributions}

We describe a very simple approach which bypasses many of the difficulties encountered in 3D face reconstruction by using a novel volumetric representation of the 3D facial geometry, and an appropriate CNN architecture that is trained to regress directly from a $2 \mathrm{D}$ facial image to the corresponding 3D volume. An overview of our method is shown in Fig. 4. In summary, our contributions are:

- Given a dataset consisting of 2D images and 3D face scans, we investigate whether a CNN can learn directly, in an end-to-end fashion, the mapping from image pixels to the full 3D facial structure geometry (including the non-visible facial parts). Indeed, we show that the answer to this question is positive.

- We demonstrate that our CNN works with just a single 2D facial image, does not require accurate alignment nor establishes dense correspondence between images, works for arbitrary facial poses and expressions, and can be used to reconstruct the whole 3D facial geometry bypassing the construction (during training) and fitting (during testing) of a 3DMM.

- We achieve this via a simple CNN architecture that performs direct regression of a volumetric representation of the 3D facial geometry from a single 2D image. 3DMM fitting is not used. Our method uses only 2D images as input to the proposed CNN architecture.

- We show how the related task of 3D facial landmark localisation can be incorporated into the proposed framework and help improve reconstruction quality, especially for the cases of large poses and facial expressions.

- We report results for a large number of experiments on both controlled and completely unconstrained images from the Web, illustrating that our method outperforms prior work on single image 3D face reconstruction by a large margin.

\section{Closely related work}

This section reviews closely related work in 3D face reconstruction, depth estimation using CNNs and work on 3D representation modelling with CNNs.

3D face reconstruction. A full literature review of 3D face reconstruction falls beyond the scope of the paper; we simply note that our method makes minimal assumptions i.e. it requires just a single $2 \mathrm{D}$ image to reconstruct the full 3D facial structure, and works under arbitrary poses and expressions. Under the single image setting, the most related works to our method are based on 3DMM fitting $[2,20,28,9,8]$ and the work of [13] which performs joint face reconstruction and alignment, reconstructing however a neutral frontal face.

The work of [20] describes a multi-feature based approach to 3DMM fitting using non-linear least-squares optimization (Levenberg-Marquardt), which given appropriate initialisation produces results of good accuracy. More recent work has proposed to estimate the update for the 3DMM parameters using CNN regression, as opposed to non-linear optimization. In [9], the 3DMM parameters are estimated in six steps each of which employs a different 
CNN. Notably, [9] estimates the 3DMM parameters on a sparse set of landmarks, i.e. the purpose of [9] is 3D face alignment rather than face reconstruction. The method of [28] is currently considered the state-of-the-art in 3DMM fitting. It is based on a single CNN that is iteratively applied to estimate the model parameters using as input the 2D image and a 3D-based representation produced at the previous iteration. Finally, a state-of-the-art cascaded regression landmark-based 3DMM fitting method is proposed in [8].

Our method is different from the aforementioned methods in the following ways:

- Our method is direct. It does not estimate 3DMM parameters and, in fact, it completely bypasses the fitting of a 3DMM. Instead, our method directly produces a 3D volumetric representation of the facial geometry.

- Because of this fundamental difference, our method is also radically different in terms of the $\mathrm{CNN}$ architecture used: we used one that is able to make spatial predictions at a voxel level, as opposed to the networks of [28, 9] which holistically predict the 3DMM parameters.

- Our method is capable of producing reconstruction results for completely unconstrained facial images from the web covering the full spectrum of facial poses with arbitrary facial expression and occlusions. When compared to the state-of-the-art CNN method for 3DMM fitting of [28], we report large performance improvement.

Compared to works based on shape from shading [10, 23], our method cannot capture such fine details. However, we believe that this is primarily a problem related to the dataset used rather than of the method. Given training data like the one produced by $[10,23]$, then we believe that our method has the capacity to learn finer facial details, too.

CNN-based depth estimation. Our work has been inspired by the work of $[5,6]$ who showed that a CNN can be directly trained to regress from pixels to depth values using as input a single image. Our work is different from [5, 6] in 3 important respects: Firstly, we focus on faces (i.e. deformable objects) whereas $[5,6]$ on general scenes containing mainly rigid objects. Secondly, [5, 6] learn a mapping from $2 \mathrm{D}$ images to $2 \mathrm{D}$ depth maps, whereas we demonstrate that one can actually learn a mapping from $2 \mathrm{D}$ to the full $3 \mathrm{D}$ facial structure including the non-visible part of the face. Thirdly, [5, 6] use a multi-scale approach by processing images from low to high resolution. In contrast, we process faces at fixed scale (assuming that this is provided by a face detector), but we build our CNN based on a state-of-the-art bottom-up top-down module [15] that allows analysing and combining CNN features at different resolutions for eventually making predictions at voxel level.

Recent work on 3D. We are aware of only one work which regresses a volume using a CNN. The work of [4] uses an LSTM to regress the 3D structure of multiple ob- ject classes from one or more images. This is different from our work in at least two ways. Firstly, we treat our reconstruction as a semantic segmentation problem by regressing a volume which is spatially aligned with the image. Secondly, we work from only one image in one single step, regressing a much larger volume of $192 \times 192 \times 200$ as opposed to the $32 \times 32 \times 32$ used in [4]. The work of [26] decomposes an input 3D shape into shape primitives which along with a set of parameters can be used to re-assemble the given shape. Given the input shape, the goal of [26] is to regress the shape primitive parameters which is achieved via a CNN. The method of [16] extends classical work on heatmap regression [24, 18] by proposing a 4D representation for regressing the location of sparse 3D landmarks for human pose estimation. Different from [16], we demonstrate that a $3 \mathrm{D}$ volumetric representation is particular effective for learning dense 3D facial geometry. In terms of 3DMM fitting, very recent work includes [19] which uses a CNN similar to the one of [28] for producing coarse facial geometry but additionally includes a second network for refining the facial geometry and a novel rendering layer for connecting the two networks. Another recent work is [25] which uses a very deep CNN for 3DMM fitting.

\section{Method}

This section describes our framework including the proposed data representation used.

\subsection{Dataset}

Our aim is to regress the full 3D facial structure from a $2 \mathrm{D}$ image. To this end, our method requires an appropriate dataset consisting of 2D images and 3D facial scans. As our target is to apply the method on completely unconstrained images from the Web, we chose the dataset of [28] for forming our training and test sets. The dataset has been produced by fitting a 3DMM built from the combination of the Basel [17] and FaceWarehouse [3] models to the unconstrained images of the $300 \mathrm{~W}$ dataset [22] using the multi-feature fitting approach of [20], careful initialisation and by constraining the solution using a sparse set of landmarks. Face profiling is then used to render each image to 10-15 different poses resulting in a large scale dataset (more than 60,000 2D facial images and 3D meshes) called 300W-LP. Note that because each mesh is produced by a 3DMM, the vertices of all produced meshes are in dense correspondence; however this is not a prerequisite for our method and unregistered raw facial scans could be also used if available (e.g. the BU-4DFE dataset [27]).

\subsection{Proposed volumetric representation}

Our goal is to predict the coordinates of the $3 \mathrm{D}$ vertices of each facial scan from the corresponding 2D image via $\mathrm{CNN}$ regression. As a number of works have pointed out 


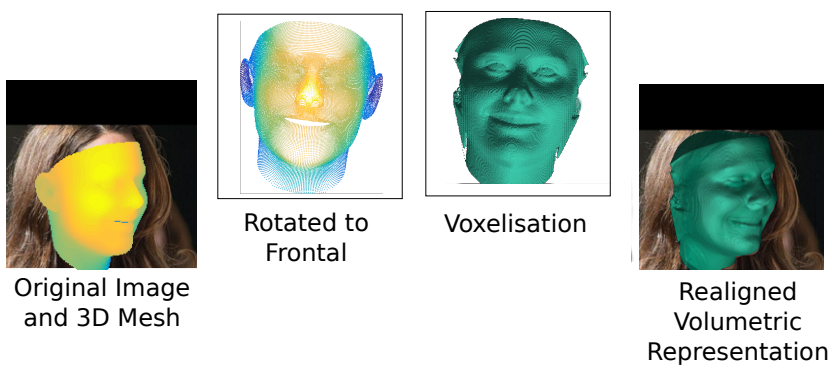

Figure 2: The voxelisation process creates a volumetric representation of the 3D face mesh, aligned with the 2D image.

(see for example [24, 18]), direct regression of all 3D points concatenated as a vector using the standard L2 loss might cause difficulties in learning because a single correct value for each 3D vertex must be predicted. Additionally, such an approach requires interpolating all scans to a vector of a fixed dimension, a pre-processing step not required by our method. Note that similar learning problems are encountered when a CNN is used to regress model parameters like the 3DMM parameters rather than the actual vertices. In this case, special care must be taken to weight parameters appropriately using the Mahalanobis distance or in general some normalisation method, see for example [28]. We compare the performance of our method with that of a similar method [28] in Section 4.

To alleviate the aforementioned learning problem, we propose to reformulate the problem of $3 \mathrm{D}$ face reconstruction as one of 2D to 3D image segmentation: in particular, we convert each 3D facial scan into a 3D binary volume $\mathbf{V}_{w h d}$ by discretizing the 3D space into voxels $\{w, h, d\}$, and then assigning the value of 1 to all points enclosed by the 3D facial scan, and 0 otherwise. That is $V_{w h d}$ is the ground truth for voxel $\{w, h, d\}$ and is equal to 1 , if voxel $\{w, h, d\}$ belongs to the $3 \mathrm{D}$ volumetric representation of the face and 0 otherwise (i.e. it belongs to the background). The conversion is shown in Fig. 2. Notice that the process creates a volume fully aligned with the $2 \mathrm{D}$ image. The importance of spatial alignment is analysed in more detail in Section 5 . The error caused by discretization for a randomly picked facial scan as a function of the volume size is shown in Fig. 3. Given that the error of state-of-the-art methods $[21,13]$ is of the order of a few mms, we conclude that discretization by $192 \times 192 \times 200$ produces negligible error.

Given our volumetric facial representation, the problem of regressing the 3D coordinates of all vertices of a facial scan is reduced to one of 3D binary volume segmentation. We approach this problem using recent CNN architectures from semantic image segmentation [14] and their extensions [15], as described in the next subsection.

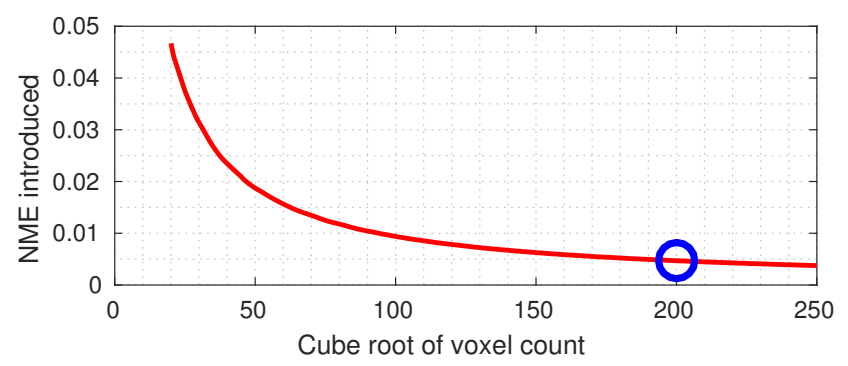

Figure 3: The error introduced due to voxelisation, shown as a function of volume density.

\subsection{Volumetric Regression Networks}

In this section, we describe the proposed volumetric regression network, exploring several architectural variations described in detail in the following subsections:

Volumetric Regression Network (VRN). We wish to learn a mapping from the 2D facial image to its corresponding 3D volume $f: \mathbf{I} \rightarrow \mathbf{V}$. Given the training set of 2D images and constructed volumes, we learn this mapping using a CNN. Our CNN architecture for 3D segmentation is based on the "hourglass network" of [15] an extension of the fully convolutional network of [14] using skip connections and residual learning [7]. Our volumetric architecture consists of two hourglass modules which are stacked together without intermediate supervision. The input is an RGB image and the output is a volume of $192 \times 192 \times 200$ of real values. This architecture is shown in Fig. 4a. As it can be observed, the network has an encoding/decoding structure where a set of convolutional layers are firstly used to compute a feature representation of fixed dimension. This representation is further processed back to the spatial domain, re-establishing spatial correspondence between the input image and the output volume. Features are hierarchically combined from different resolutions to make per-pixel predictions. The second hourglass is used to refine this output, and has an identical structure to that of the first one.

We train our volumetric regression network using the sigmoid cross entropy loss function:

$l_{1}=\sum_{w=1}^{W} \sum_{h=1}^{H} \sum_{d=1}^{D}\left[V_{w h d} \log \widehat{V}_{w h d}+\left(1-V_{w h d}\right) \log \left(1-\widehat{V}_{w h d}\right)\right]$,

where $\widehat{V}_{w h d}$ is the corresponding sigmoid output at voxel $\{w, h, d\}$ of the regressed volume.

At test time, and given an input 2D image, the network regresses a 3D volume from which the outer 3D facial mesh is recovered. Rather than making hard (binary) predictions at pixel level, we found that the soft sigmoid output is more useful for further processing. Both representations are shown in Fig. 5 where clearly the latter results in smoother results. Finally, from the 3D volume, a mesh can be formed 


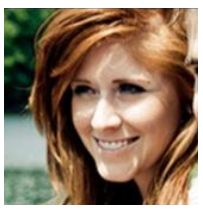

Input
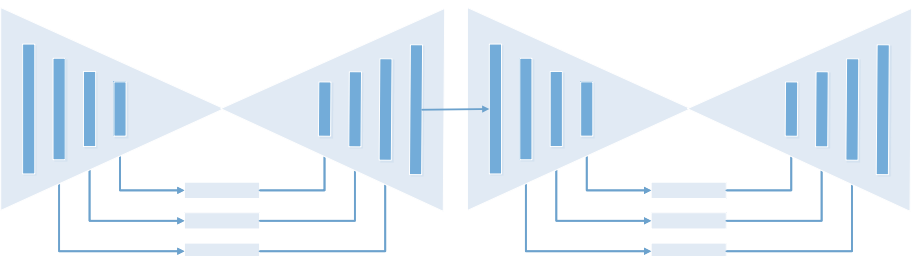

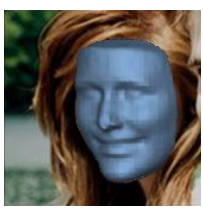

Output

(a) The proposed Volumetric Regression Network (VRN) accepts as input an RGB input and directly regresses a 3D volume completely bypassing the fitting of a 3DMM. Each rectangle is a residual module of 256 features.

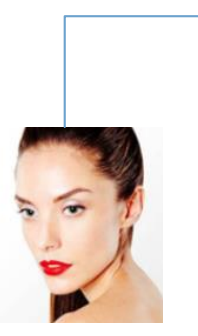

Input
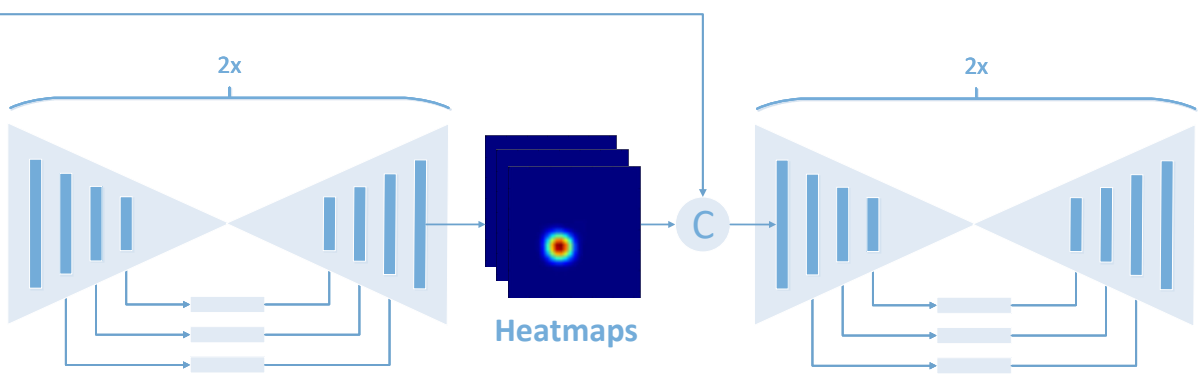

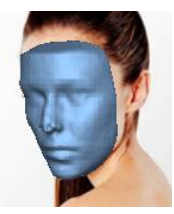

Output

(b) The proposed VRN - Guided architecture firsts detects the 2D projection of the 3D landmarks, and stacks these with the original image. This stack is fed into the reconstruction network, which directly regresses the volume.

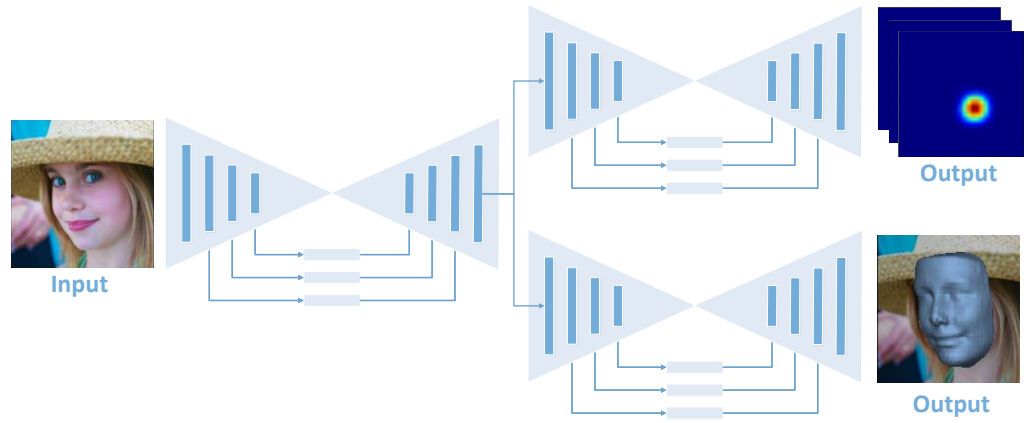

(c) The proposed VRN - Multitask architecture regresses both the 3D facial volume and a set of sparse facial landmarks.

Figure 4: An overview of the proposed three architectures for Volumetric Regression: Volumetric Regression Network (VRN), VRN - Guided and VRN - Multitask.

by generating the iso-surface of the volume. If needed, correspondence between this variable length mesh and a fixed mesh can be found using Iterative Closest Point (ICP).

VRN - Multitask. We also propose a Multitask VRN, shown in Fig. 4c, consisting of three hourglass modules. The first hourglass provides features to a fork of two hourglasses. The first of this fork regresses the 68 iBUG landmarks [22] as 2D Gaussians, each on a separate channel. The second hourglass of this fork directly regresses the 3D structure of the face as a volume, as in the aforementioned unguided volumetric regression method. The goal of this multitask network is to learn more reliable features which are better suited to the two tasks.

VRN - Guided. We argue that reconstruction should benefit from firstly performing a simpler face analysis task; in particular we propose an architecture for volumetric regression guided by facial landmarks. To this end, we train
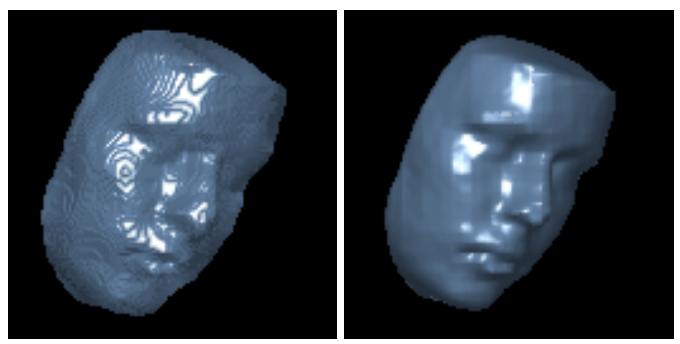

Figure 5: Comparison between making hard (binary) vs soft (real) predictions. The latter produces a smoother result.

a stacked hourglass network which accepts guidance from landmarks during training and inference. This network has a similar architecture to the unguided volumetric regression method, however the input to this architecture is an RGB image stacked with 68 channels, each containing a Gaussian ( $\sigma=1$, approximate diameter of 6 pixels) centred on each 


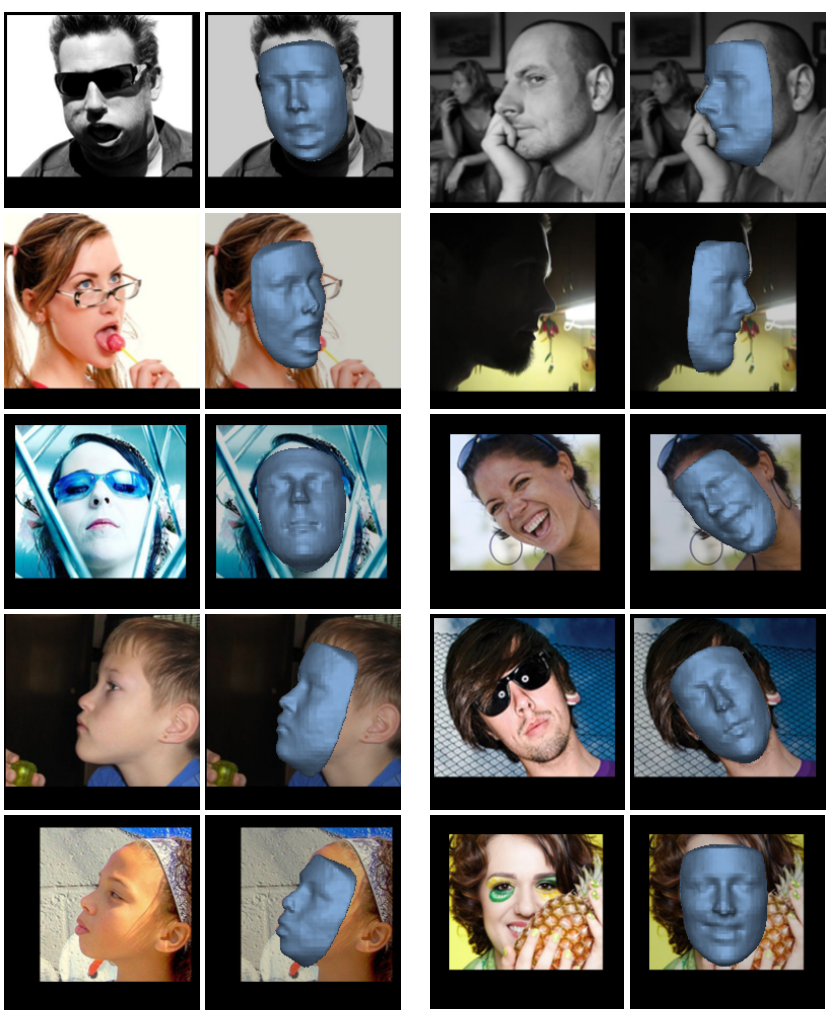

Figure 6: Some visual results from the AFLW2000-3D dataset generated using our VRN - Guided method.

of the 68 landmarks. This stacked representation and architecture is demonstrated in Fig. 4b. During training we used the ground truth landmarks while during testing we used a stacked hourglass network trained for facial landmark localisation. We call this network VRN - Guided.

\subsection{Training}

Each of our architectures was trained end-to-end using RMSProp with an initial learning rate of $10^{-4}$, which was lowered after 40 epochs to $10^{-5}$. During training, random augmentation was applied to each input sample (face image) and its corresponding target (3D volume): we applied in-plane rotation $r \in\left\{-45^{\circ}, \ldots, 45^{\circ}\right\}$, translation $t_{z}, t_{y} \in\{-15, \ldots, 15\}$ and scale $s \in 1-\{-0.15, \ldots, 0.15\}$ jitter. In $20 \%$ of cases, the input and target were flipped horizontally. Finally, the input samples were adjusted with some colour scaling on each RGB channel.

In the case of the VRN - Guided, the landmark detection module was trained to regress Gaussians with standard deviation of approximately 3 pixels $(\sigma=1)$.

\section{Results}

We performed cross-database experiments only, on 3 different databases, namely AFLW2000-3D, BU-4DFE, and Florence reporting the performance of all the proposed
Table 1: Reconstruction accuracy on AFLW2000-3D, BU4DFE and Florence in terms of NME. Lower is better.

\begin{tabular}{|l||c|c|c|}
\hline Method & AFLW2000-3D & BU-4DFE & Florence \\
\hline \hline VRN & 0.0676 & 0.0600 & 0.0568 \\
VRN - Multitask & 0.0698 & 0.0625 & 0.0542 \\
VRN - Guided & $\mathbf{0 . 0 6 3 7}$ & $\mathbf{0 . 0 5 5 5}$ & $\mathbf{0 . 0 5 0 9}$ \\
\hline 3DDFA [28] & 0.1012 & 0.1227 & 0.0975 \\
EOS [8] & 0.0971 & 0.1560 & 0.1253 \\
\hline
\end{tabular}

networks (VRN, VRN - Multitask and VRN - Guided) along with the performance of two state-of-the-art methods, namely 3DDFA [28] and EOS [8]. Both methods perform 3DMM fitting (3DDFA uses a CNN), a process completely bypassed by $V R N$.

Our results can be found in Table 1 and Figs. 7 and 8. Visual results of the proposed VRN - Guided on some very challenging images from AFLW2000-3D can be seen in Fig. 6. Examples of failure cases along with a visual comparison between $V R N$ and $V R N$ - Guided can be found in the supplementary material. From these results, we can conclude the following:

1. Volumetric Regression Networks largely outperform 3DDFA and EOS on all datasets, verifying that directly regressing the $3 \mathrm{D}$ facial structure is a much easier problem for CNN learning.

2. All VRNs perform well across the whole spectrum of facial poses, expressions and occlusions. Also, there are no significant performance discrepancies across different datasets (ALFW2000-3D seems to be slightly more difficult).

3. The best performing VRN is the one guided by detected landmarks (VRN - Guided), however at the cost of higher computational complexity: VRN - Guided uses another stacked hourglass network for landmark localization.

4. VRN - Multitask does not always perform particularly better than the plain VRN (in fact on BU-4DFE it performs worse), not justifying the increase of network complexity. It seems that it might be preferable to train a network to focus on the task in hand.

Details about our experiments are as follows:

Datasets. (a) AFLW2000-3D: As our target was to test our network on totally unconstrained images, we firstly conducted experiments on the AFLW2000-3D [28] dataset which contains 3D facial meshes for the first 2000 images from AFLW [12]. (b) BU-4DFE: We also conducted experiments on rendered images from BU-4DFE [27]. We rendered each participant for both Happy and Surprised expressions with three different pitch rotations between -20 and 20 degrees. For each pitch, seven roll rotations from -80 to 80 degrees were also rendered. Large variations in lighting direction and colour were added randomly to make the images more challenging. (c) Florence: Finally, we 

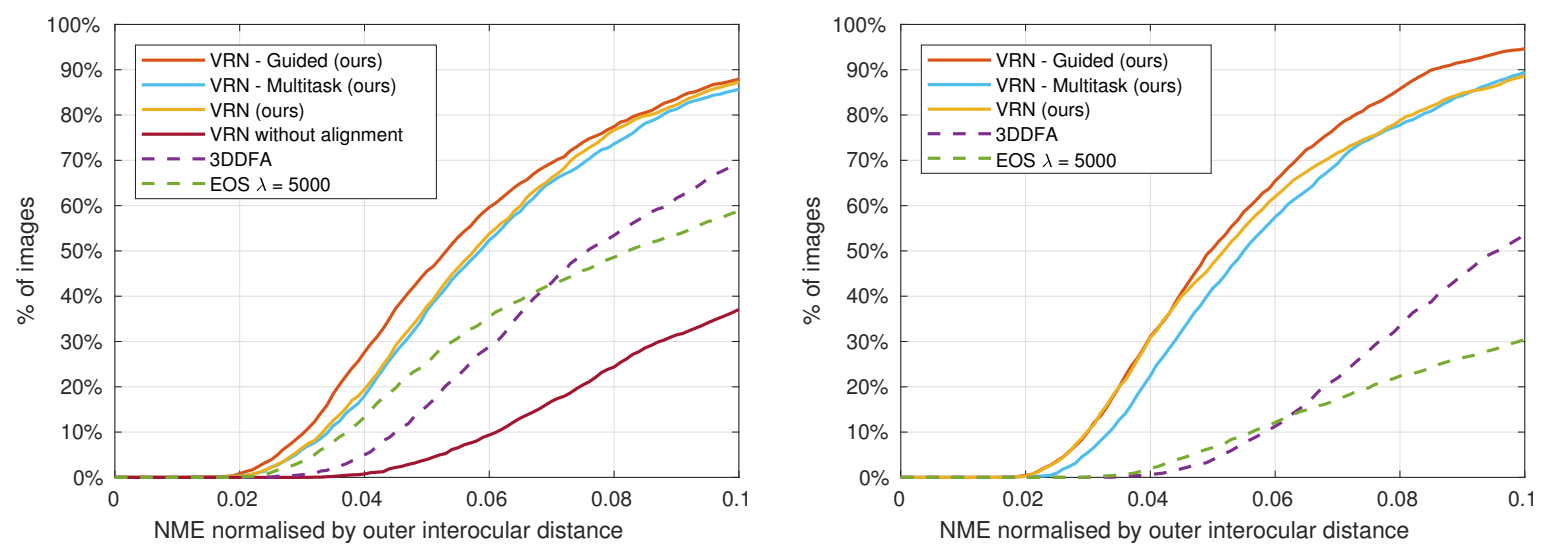

Figure 7: NME-based performance on in-the-wild ALFW2000-3D dataset (left) and renderings from BU-4DFE (right). The proposed Volumetric Regression Networks, and EOS and 3DDFA are compared.

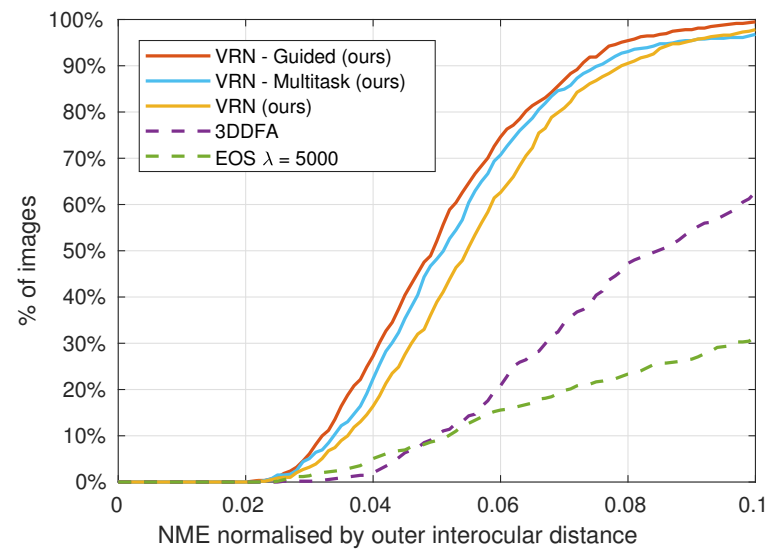

Figure 8: NME-based performance on our large pose renderings of the Florence dataset. The proposed Volumetric Regression Networks, and EOS and 3DDFA are compared.

conducted experiments on rendered images from the Florence [1] dataset. Facial images were rendered in a similar fashion to the ones of BU-4DFE but for slightly different parameters: Each face is rendered in 20 difference poses, using a pitch of $-15,20$ or 25 degrees and each of the five evenly spaced rotations between -80 and 80 .

Error metric. To measure the accuracy of reconstruction for each face, we used the Normalised Mean Error (NME) defined as the average per vertex Euclidean distance between the estimated and ground truth reconstruction normalised by the outer 3D interocular distance:

$$
\mathrm{NME}=\frac{1}{N} \sum_{k=1}^{N} \frac{\left\|\mathbf{x}_{k}-\mathbf{y}_{k}\right\|_{2}}{d},
$$

where $N$ is the number of vertices per facial mesh, $d$ is the 3D interocular distance and $\mathbf{x}_{k}, \mathbf{y}_{k}$ are vertices of the grouthtruth and predicted meshes. The error is calculated on the face region only on approximately 19,000 vertices per facial mesh. Notice that when there is no point correspondence between the ground truth and the estimated mesh, ICP was used but only to establish the correspondence, i.e. the rigid alignment was not used. If the rigid alignment is used, we found that, for all methods, the error decreases but it turns out that the relative difference in performance remains the same. For completeness, we included these results in the supplementary material.

Comparison with state-of-the-art. We compared against state-of-the-art 3D reconstruction methods for which code is publicly available. These include the very recent methods of [28], called 3DDFA, and [8], called EOS ${ }^{1}$.

\section{Importance of spatial alignment}

The 3D reconstruction method described in [4] regresses a 3D volume of fixed orientation from one or more images using an LSTM. This is different to our approach of taking a single image and regressing a spatially aligned volume, which we believe is easier to learn. To explore what the repercussions of ignoring spatial alignment are, we trained a variant of $V R N$ which regresses a frontal version of the face, i.e. a face of fixed orientation as in $[4]^{2}$.

Although this network produces a reasonable face, it is capable of capturing diminished expression, and the shape for all faces appears to remain almost identical. This is very noticeable in Fig. 9. We also show a numeric comparison in Fig. 7 (left), as VRN without alignment. We believe that this further confirms that spatial alignment is of paramount importance when performing $3 \mathrm{D}$ reconstruction in this way.

\footnotetext{
${ }^{1}$ For EOS we used a large regularisation parameter $\lambda=5000$ which we found to offer the best performance for most images. The method uses $2 \mathrm{D}$ landmarks as input, so for the sake of a fair comparison a stacked hourglass for 2D landmark detection was trained for this purpose. Our tests were performed using v0.12 of EOS.

${ }^{2}$ We also attempted to train a network using the code from [4] on downsampled versions of our own volumes. Unfortunately, we were unable to get the network to learn anything.
} 

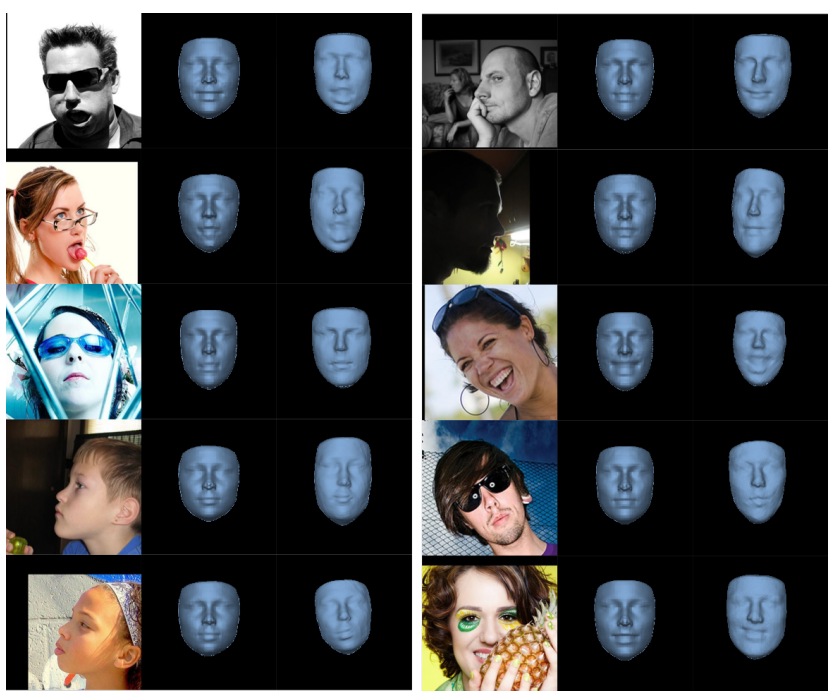

Figure 9: Result from VRN without alignment (second columns), and a frontalised output from VRN - Guided (third columns).

\section{Ablation studies}

In this section, we report the results of experiments aiming to shed further light into the performance of the proposed networks. For all experiments reported, we used the best performing VRN - Guided.

Effect of pose. To measure the influence of pose on the reconstruction error, we measured the NME for different yaw angles using all of our Florence [1] renderings. As shown in Fig. 10, the performance of our method decreases as the pose increases. This is to be expected, due to less of the face being visible which makes evaluation for the invisible part difficult. We believe that our error is still very low considering these poses.

Effect of expression. Certain expressions are usually considered harder to accurately reproduce in 3D face reconstruction. To measure the effect of facial expressions on performance, we rendered frontal images in difference expressions from BU-4DFE (since Florence only exhibits a neutral expression) and measured the performance for each expression. This kind of extreme acted facial expressions generally do not occur in the training set, yet as shown in Fig. 11, the performance variation across different expressions is quite minor.

Effect of Gaussian size for guidance. We trained a $V R N$ - Guided, however, this time, the facial landmark detector network of the VRN - Guided regresses larger Gaussians ( $\sigma=2$ as opposed to the normal $\sigma=1$ ). The performance of the $3 \mathrm{D}$ reconstruction dropped by a negligible amount, suggesting that as long as the Gaussians are of a sensible size, guidance will always help.

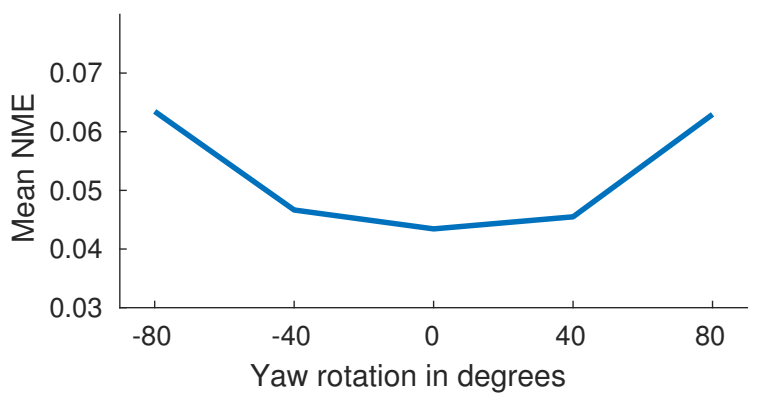

Figure 10: The effect of pose on reconstruction accuracy in terms of NME on the Florence dataset. The VRN - Guided network was used.

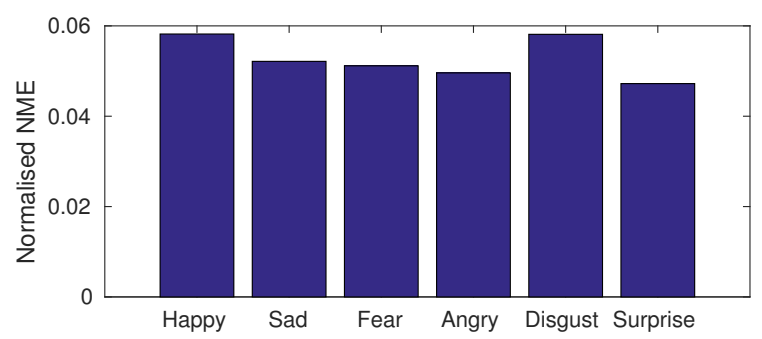

Figure 11: The effect of facial expression on reconstruction accuracy in terms of NME on the BU-4DFE dataset. The $V R N$ - Guided network was used.

\section{Conclusions}

We proposed a direct approach to 3D facial reconstruction from a single 2D image using volumetric CNN regression. To this end, we proposed and exhaustively evaluated three different networks for volumetric regression, reporting results that show that the proposed networks perform well for the whole spectrum of facial pose, and can deal with facial expressions as well as occlusions. We also compared the performance of our networks against that of recent state-of-the-art methods based on 3DMM fitting reporting large performance improvement on three different datasets. Future work may include improving detail and establishing a fixed correspondence from the isosurface of the mesh.

\section{Acknowledgements}

Aaron Jackson is funded by a PhD scholarship from the University of Nottingham. We are grateful for access to the University of Nottingham High Performance Computing Facility. Finally, we would like to express our thanks to Patrik Huber for his help testing EOS [8]. 


\section{References}

[1] A. D. "Bagdanov, I. Masi, and A. Del Bimbo. The florence $2 \mathrm{~d} / 3 \mathrm{~d}$ hybrid face datset. In Proc. of ACM Multimedia Int.l Workshop on Multimedia access to $3 D$ Human Objects (MA3HO11). ACM, ACM Press, December 2011. 7, 8

[2] V. Blanz and T. Vetter. A morphable model for the synthesis of $3 \mathrm{~d}$ faces. In Computer graphics and interactive techniques, 1999. 2

[3] C. Cao, Y. Weng, S. Zhou, Y. Tong, and K. Zhou. Facewarehouse: A $3 \mathrm{~d}$ facial expression database for visual computing. IEEE TVCG, 20(3), 2014. 3

[4] C. B. Choy, D. Xu, J. Gwak, K. Chen, and S. Savarese. 3dr2n2: A unified approach for single and multi-view $3 \mathrm{~d}$ object reconstruction. arXiv preprint arXiv:1604.00449, 2016. 3, 7

[5] D. Eigen and R. Fergus. Predicting depth, surface normals and semantic labels with a common multi-scale convolutional architecture. In ICCV, 2015. 3

[6] D. Eigen, C. Puhrsch, and R. Fergus. Depth map prediction from a single image using a multi-scale deep network. In NIPS, 2014. 3

[7] K. He, X. Zhang, S. Ren, and J. Sun. Deep residual learning for image recognition. 2016. 4

[8] P. Huber, G. Hu, R. Tena, P. Mortazavian, W. P. Koppen, W. Christmas, M. Rätsch, and J. Kittler. A multiresolution 3d morphable face model and fitting framework. 2, 3, 6, 7, 8

[9] A. Jourabloo and X. Liu. Large-pose face alignment via cnnbased dense $3 \mathrm{~d}$ model fitting. In CVPR, 2016. 2, 3

[10] I. Kemelmacher-Shlizerman and R. Basri. 3d face reconstruction from a single image using a single reference face shape. IEEE TPAMI, 33(2):394-405, 2011. 2, 3

[11] I. Kemelmacher-Shlizerman and S. M. Seitz. Face reconstruction in the wild. In ICCV, 2011. 2

[12] M. Koestinger, P. Wohlhart, P. M. Roth, and H. Bischof. Annotated facial landmarks in the wild: A large-scale, realworld database for facial landmark localization. In First IEEE International Workshop on Benchmarking Facial Image Analysis Technologies, 2011. 6

[13] F. Liu, D. Zeng, Q. Zhao, and X. Liu. Joint face alignment and $3 \mathrm{~d}$ face reconstruction. In ECCV, 2016. 2, 4

[14] J. Long, E. Shelhamer, and T. Darrell. Fully convolutional networks for semantic segmentation. In CVPR, 2015. 4

[15] A. Newell, K. Yang, and J. Deng. Stacked hourglass networks for human pose estimation. In ECCV, 2016. 3, 4

[16] G. Pavlakos, X. Zhou, K. G. Derpanis, and K. Daniilidis. Coarse-to-fine volumetric prediction for single-image $3 \mathrm{~d}$ human pose. arXiv preprint arXiv:1611.07828, 2016. 3

[17] P. Paysan, R. Knothe, B. Amberg, S. Romdhani, and T. Vetter. A $3 \mathrm{~d}$ face model for pose and illumination invariant face recognition. In AVSS, 2009. 3

[18] T. Pfister, J. Charles, and A. Zisserman. Flowing convnets for human pose estimation in videos. In ICCV, 2015. 3, 4

[19] E. Richardson, M. Sela, R. Or-El, and R. Kimmel. Learning detailed face reconstruction from a single image. arXiv preprint arXiv:1611.05053, 2016. 3

[20] S. Romdhani and T. Vetter. Estimating 3d shape and texture using pixel intensity, edges, specular highlights, texture constraints and a prior. In $C V P R, 2005.2,3$
[21] J. Roth, Y. Tong, and X. Liu. Adaptive 3d face reconstruction from unconstrained photo collections. In CVPR, 2016. 2, 4

[22] C. Sagonas, G. Tzimiropoulos, S. Zafeiriou, and M. Pantic. A semi-automatic methodology for facial landmark annotation. In $C V P R-W, 2013.3,5$

[23] S. Suwajanakorn, I. Kemelmacher-Shlizerman, and S. M. Seitz. Total moving face reconstruction. In ECCV, 2014. 2,3

[24] J. Tompson, R. Goroshin, A. Jain, Y. LeCun, and C. Bregler. Efficient object localization using convolutional networks. In CVPR, 2015. 3, 4

[25] A. T. Tran, T. Hassner, I. Masi, and G. Medioni. Regressing robust and discriminative $3 \mathrm{~d}$ morphable models with a very deep neural network. arXiv preprint arXiv:1612.04904, 2016. 3

[26] S. Tulsiani, H. Su, L. J. Guibas, A. A. Efros, and J. Malik. Learning shape abstractions by assembling volumetric primitives. arXiv preprint arXiv:1612.00404, 2016. 3

[27] L. Yin, X. Chen, Y. Sun, T. Worm, and M. Reale. A highresolution 3d dynamic facial expression database. In Automatic Face \& Gesture Recognition, 2008. FG'08. 8th IEEE International Conference on, pages 1-6. IEEE, 2008. 3, 6

[28] X. Zhu, Z. Lei, X. Liu, H. Shi, and S. Z. Li. Face alignment across large poses: A 3d solution. 2016. 2, 3, 4, 6, 7 